\title{
THE NONLINEAR NATURE OF PREFERENCES, ITS IMPACT ON THE SENSITIVITY AND EFFECTIVENESS OF MULTIPLE CRITERIA ALTERNATIVES
}

\author{
Rafael Sarkisyan \\ Moscow State University of Railway Engineering \\ E-mail: sarkisyanry@mail.ru \\ Aleksandra Masalida \\ Moscow State University of Railway Engineering \\ masalido4ka@rambler.ru \\ Elena Kobets \\ Moscow State University of Railway Engineering \\ elenavk2706@mail.ru
}

\begin{abstract}
The mechanisms of creating and processing expert and/or statistical information, which underlie the $A H P / A N P$, do not enable to take into account the nonlinear nature of preferences and their dependence upon intensiveness (or level) of measurable features and qualities of researched and optimized systems. In the traditional methods of multiple criteria (multiobjective) optimization based on the concept of preferences and utilities, the nonlinear nature of preferences as well as the loss of sensitivity and effectiveness of alternatives caused by it can be taken into account, if to proceed from the concave increasing property of a corresponding evaluation function (preference function). If to assume also that the concave increasing preference function $u: E^{m} \rightarrow E^{1} \quad F_{0} \subset E^{m}$ is differentiable on the nonempty convex set of estimates

$$
u(f)-u(\bar{f}) \leq \nabla u(\bar{f})^{T}(f-\bar{f}), \quad f \in F_{0}
$$

then from its peculiar differential inequality

$$
u(f)=u_{0}(f)+\nabla u(f)^{T} f, f \in F_{0} .
$$

one can deduce the decomposition scheme

$$
u_{0}(f)
$$

where is the height of intersection of the hyperplane $H_{u}=\left\{(y, f) / y=u(\bar{f})+\nabla u(\bar{f})^{T}(f-\bar{f}), f \in F_{0}\right\}$

, tangential to the surface of

$$
u(f) \quad \bar{f}, \quad \nabla u(\bar{f})
$$

the function , with the axle of the latter at the point and is the gradient of the function. The second component of the decomposition, $v(f)=\nabla u(f)^{T} f$,

$$
\begin{gathered}
\sigma(f) \equiv \lim _{t \rightarrow 1} \frac{t}{u(t f)} \frac{d u(t f)}{d t} \\
=\frac{1}{u(f)} \sum_{j=1}^{m} \frac{\partial u(f)}{\partial f_{j}} f_{j}
\end{gathered}
$$
\end{abstract}




$$
\begin{aligned}
& v(f)=\sum_{j=1}^{m} \frac{\partial u(f)}{\partial f_{j}} f_{j}=\sigma(f) u(f) \\
& \quad \text { "sensitive" component } u(f) \\
& v_{j}(f)=\frac{\partial u(f)}{\partial f_{j}} f_{j}=\sigma_{j}(f) u(f), j=1, \ldots, m
\end{aligned}
$$

, in effect, characterize the relative weight (importance, return) of individual criteria and play the same role in

$$
\omega=\left(\omega_{1}, \omega_{2}, \ldots, \omega_{m}\right)^{T}
$$

alternatives ranking as the eigenvector coordinates

do in the AHP/ANP schemes, but now they depend on the state vector. Their ratio, $v_{i}(f) / v_{j}(f)=\sigma_{i}(f) / \sigma_{j}(f)=\left(f_{i} / f_{j}\right) \mu_{i j}, i, j=1, \ldots, m$,

where

$\mu_{i j}, i, j=1, \ldots, m$

are marginal rates of substitution between the criteria, allows to

$$
f_{i} / f_{j}=1 / \mu_{i j}=\mu_{j i} \text {. }
$$

find stable solutions that would satisfy the condition The

$$
\begin{aligned}
& \text { maximization of the functions } \begin{aligned}
v(f) & \sigma(f) \\
\text { and } & f^{c}(\lambda) \quad \lambda \leq 1,
\end{aligned} \text { on the surface of } \\
& \text { generates a trajectory of solutions } \quad, \quad \text { that satisfy both the maximum of }
\end{aligned}
$$
the characteristics $v\left(f^{c}\right)$ and $\sigma(f)$, and a "consistent" value of the criteria

$$
\theta\left(f^{c}\right)=-f^{c T} H f^{c}=(1-\lambda) v\left(f^{c}\right)=(1-\lambda) c \sigma\left(f^{c}\right)
$$

interaction function

$\lambda \leq 1$

$$
\text { , where }
$$

is the Lagrange multiplier, $H$ is the Hessian matrix of the function .

Also the possibility of using the proposed analytic correlations in the AHP/ANP traditional procedures is being discussed. As applications a multiple criteria problem of corporate resources management and diagnostic messages processing in transport systems are being considered.

Keywords: preferences, nonlinear nature, sensitivity, effectiveness of alternatives, optimization on surface, trajectory, stability.

\section{Introduction}

Applied multiple criteria problems and interactive methods of their solution serve as the context of the proposed study. Traditionally, one of the important streams of scholarship in this field of knowledge was based on the concept of preferences and utilities that show up on the set of estimates of alternatives by criteria. Experience and intuition suggest that preferences are of nonlinear nature which leads to the loss of sensitivity and effectiveness of causal relations. The necessity to describe analytically these nonlinear effects and to take them into account in choice and decision making procedures acted as a spur to the present study. Involving the theory of sensitivity allows to construct suitable analytic tools (measures of relative sensitivity and effectiveness for nonlinear causal relations) contributory to ranking solutions according to their preference. The goal is a more adequate description of preferences, 
raise of accuracy and utility of sought-for solutions and of actions planned on their base.

\section{Literature Review}

As far as we know, the idea of spatio-temporal dependence of preferences was first stated in (Keeney R., \& Raiffa H., 1976). In effect, the logic of constructing effective man - machine methods and procedures for solving practical multiple criteria problems also proceeds from an assumption of dependence of preferences upon an (achieved) level of criteria (Benayoun R., 1971; Hall A.D., 1962; Fandel G., \& Wilhelm J., 1976; Geoffrion, A.M., 1972). To develop the ideas of the authors (Geoffrion, A.M., 1972), in (Sarkisyan R., 1992) the possibility was substantiated of representing the relative importance of criteria via the ratio of directional derivatives of criterion functions. The results of some recent studies (DellaVigna S., 2009; Hands W., 2010.) also speak in favour of the dependence of preferences upon the evaluated state and upon trends of its development in time.

The approach contained in the proposal and its analytic correlations (measures of sensitivity and effectiveness, optimization on surface) that characterize the nonlinear preferences are original results of applying the theory of sensitivity to modeling nonlinear effects which show up during the formation of expert information about criteria and alternatives.

\section{Objectives and Hypotheses}

The main assumption is brought to the existence of the concave increasing twice differentiable preference function on the set of criteria values. Such a function $u(f)-u(\bar{f}) \leq \nabla u(\bar{f})^{T}(f-\bar{f}), \quad f \in F_{0}$,

satisfies the differentiable inequality and $u(f)=u_{0}(f)+\nabla u(f)^{T} f$ allows a decomposition in the form $=(1-\sigma(f)) u(f)+\sigma(f) u(f), f \in F_{0}, \quad \sigma(f)=\sigma_{1}(f)+\ldots+\sigma_{m}(f)$ where $\quad$ is the $v(f)=\nabla u(f)^{T} f=v_{1}(f)+\ldots+v_{m}(f)$

measure of relative sensitivity, and is the measure of effectiveness (effective return). The maximization of these characteristics on the preference function level surface allows to reach necessary ranking of criteria values.

\section{Research Design}

The interactive methods of multiple criteria optimization are in harmony with the spirit of the studies (Saaty T.L., 1994; Saaty T.L., 2001) aimed at finding a reasonable compromise between rivaling criteria and requirements. In this sense the hierarchy $<$ Goal, Criteria, Alternatives $>$ is inherent to any problem of optimization on the base of multiple criteria logic and serves, according to T. Saaty, as a mighty speculative model for describing and researching complex systems.

\section{Premises}

In one of the earlier studies related to researching the reliability of the computerized system TELEPERM-ME (Siemens) by means of the AHP/ANP models we came to a conclusion that the idea of a "reasonable compromise" harmonizes with that of "ideal proportions" (Geoffrion A., 1972). Such proportions can be obtained by maximizing the functions $v(f)$ and $\sigma(f)$ on the surface of $u(f)=$ const The ratios 


$$
v_{i}(f) / v_{j}(f)=\sigma_{i}(f) / \sigma_{j}(f)=\left(f_{i} / f_{j}\right) \mu_{i j}, i, j=1, \ldots, m,
$$

satisfy this quality of

solutions. Optimization on the preference function level surface also satisfies Simon's principle of satisficing (Simon H., 1979), while the role of consent index in $A H P / A N P$ is played by a relation which allows to evaluate the level of the criteria $\theta\left(f^{c}\right)=-f^{c T} H f^{c}=(1-\lambda) v\left(f^{c}\right)=(1-\lambda) c \sigma\left(f^{c}\right)$.

interaction function

This

relation is just at all points of the optimal trajectory It means that at every point of the optimal trajectory the level of the criteria interaction function is

defined by the maximum value of indicators $v(f)$ and $\sigma(f)$.

\section{Limitations}

It is assumed that during the increase of the criteria values the preference function is aiming at its established value (i.e. it is "getting sated"). In this case the measure of its relative sensitivity $\sigma(f)$ is a monotonously decreasing function, while the "sensitive" component $v(f)$ reaches its maximum on the level surface of $u(f)=$ const and, therefore, the given device can be successfully applied.

According to the Debreu theorem, if the preference relation on the set of criteria values is a complete preorder and has properties of monotonousness and of continuity, then the preference (or utility, value) function can be put in correspondence with it; the property of differentiability is entered for convenience of mathematical analysis. It is not expedient to apply the given approach unless it is assumed that the preference function is "getting sated".

\section{Conclusion}

Our professional interest in the $A H P / A N P$ technique and models is conditioned only by issues and prospects of development of interactive methods of applied multiple criteria problems solution; the application aspect is to create decision making support systems for projecting, planning and managing in technical and organizational systems.

In the research practice related to applied multiple criteria problems solution, the interactive methods and procedures play a leading role. The traditional view on the algorithmization of the preferred solutions search process proceeds from the consecutive revelation and description of preferences as a function of state and movement direction in the space of estimates and alternatives. It is accepted that namely such a multi-step (multi-stage) search process will allow to decrease uncertainty and non-comparableness and to reach a reasonable balancing (Saaty T,L., 2001) between rivaling requirements of criteria. The preferences are of nonlinear nature which generates the loss of sensitivity and effectiveness of a corresponding causal relation. The approach stated in the proposal allows to model more adequately these nonlinear effects and to take them into account while ranking multiple criteria estimates and corresponding alternative solutions. In our view, the relation of these factors to the $A H P / A N P$ is obvious.

\section{Key References}

Geoffrion, A.M., Dyer J.S., \& Fienberg A. (1972). An interactive approach for multicriterion optimization, with an application to the operation of an academic department. Management Science, 19(4), part 1, 357-368. 
Benayoun R., de Montgolfier J., Tergny J., \& Laritchev O. (1971). Linear programming with multiple objective functions: STEP method. Mathematical Programming, 1(3), 365-375.

Keeney R., \& Raiffa H. (1976). Decisions with multiple objectives: preferences and value tradeoffs. New York: John Wiley \& Sons, Inc.

Simon H. (1979). Rational decision making in business organization. American Economic Review, 69(4), 493-513.

Hall A.D. (1962). A methodology for systems engineering. Princeton: D. van Nostrand Comp., Inc.

Fandel G., \& Wilhelm J. (1976). Zur Eintscheidng Theorie bei mehrfacher Zielsetzung. Zeitschrift für Operation Research , 20. 1-20.

DellaVigna S. (2009). Psychology and economics: evidence from the field. Journal of Economic Literature, 47(2), 315-372.

Hands W. (2010). Normative rational choice theory: past, present and future. Fourth annual conference on the history of recent economic (HISRECO), Cachan, France.

Saaty T.L. (1994). Fundamentals of decision making and priority theory with the AHP, vol. 6. Pittsburgh, PA: RWS Publications.

Saaty T,L. (2001). Decision making with dependence and feedback, The analytic network process. PA: RWS Publications.

Sarkisyan R.(1992). Adaptive procedures for dialogue systems: Methods of directional derivatives. Doctoral thesis, Yerevan Polytechnic Institute.

\section{Applications}

The analytical apparatus stated in the proposal can be illustrated by the example of two applications:

a) Corporate resources management during transport systems maintenance and repair planning. On the base of regarding the technological relation between the planned work volume and costs of economic factors made for that purpose as a $\bar{y}=F\left(q_{1}, \ldots, q_{n}\right)$,

production function a bicriteria optimization problem is being formulated which includes minimization of the cost function $\left.r(q)=p_{1} q_{1}+\ldots+p_{n} q_{n}\right)$

and maximization of the return function $v(q)=\frac{\partial F}{\partial q_{1}} q_{1}+\ldots+\frac{\partial F}{\partial q_{n}} q_{n}$.

The production function, at that, is being regarded as a twice continuously differentiable concave increasing function, thus allowing a $F(q)=F_{0}(q)+v(q)=(1-\sigma(q)) F(q)+\nabla F(q)^{T} q$,

decomposition in the form

As a suitable preference function on the set of criteria values the approximations $u(f)=a^{T} f+(1 / 2) f^{T} H f, a+H f \hbar 0 \quad u(f)=U_{0}\left(1-e^{-\alpha_{1} f_{1}}\right)\left(1-e^{-\alpha_{2} f_{2}}\right)$

and $\quad$ are being considered, where the coordinates $f_{1}$ and $f_{2}$ represent corresponding criteria 
functions. Optimization on the surface $\bar{y}=F\left(q_{1}, \ldots, q_{n}\right)$ is being made using the Lagrange method.

b) Diagnostic messages processing for high speed vehicle.

As it is known, the problem of diagnostics can be considered from the point of view of a problem of choosing one of possible explanations on the base of available data. It is assumed that an observed object is exhaustively described by a set of parameters $x_{l}$, $x_{2}, \ldots, x_{n}$, which make up the description vector $\boldsymbol{x}=\left(x_{1}, \ldots, x_{n}\right)^{T} \bar{x} D$, where $D$ is the space of descriptions, the set of various values of the vector $\boldsymbol{x}$. Also it is assumed that to every state $S_{i}$ from among the set of states $S=\left\{S_{1}, S_{2}, \ldots, S_{m}\right\}$ corresponds an area $D_{i} \grave{I} D, i=1, \ldots, m$. If these areas are already established, the problem of diagnostics can be brought to decision making in favour of a state (or hypothesis) $S_{i}$ any time its description $\boldsymbol{x}$ falls on an area $D_{i}$. As the solution criteria the following are being regarded:

- maximization of the a posteriori probability function $P\left(S_{i} / x\right)=, i=1, \ldots, m$;

$$
l\left(x \in D_{i}\right),
$$

- minimization of the loss function losses being caused by a false classification.

In case of Euclidean description space, i. e. $D^{\overline{ }} E^{n}$, the criteria are interpreted in terms of corresponding probability density functions on the subsets $D_{i}, i=1, \ldots, m$.

In effect, both applications represent a hierarchical model $<$ Goal - Criteria Alternatives $>$. In the first application we deal with a continued set of alternatives presented by the level surface equation

$$
\bar{y}=F\left(q_{1}, \ldots, q_{n}\right)
$$

the set of states $\mathrm{S}=\{\mathrm{S} 1, \mathrm{~S} 2, \ldots, \mathrm{Sm}\}$ subject to identification (or classification) serves as a set of alternatives.

Note that in both cases the preferences depend upon the criteria values. In $A H P / A N P$ models the judgment matrix looks as $A=\left(a_{i j)}\right), a_{11}=a_{22}=1, a_{12}=1 / a_{21}$. The corresponding priority estimates will be equal to: $\quad$ o1 $=a_{12} /\left(1+a_{12}\right), \quad 0_{2}=1 /(1+$ $\left.a_{12}\right),{ }_{0} /{ }_{02}=a_{12}$, as follows from the model of numerical judgments processing $A$ 。 $=$ 가 $_{\max } \circ$, 과 ${ }_{\max }=2$. These estimates of relative priorities are not acceptable for both (or similar) applications, despite the efforts of experts. According to the material of the proposal, their role can be played by the measures $v_{i}(f), i=1, \ldots, m$, more precisely, by their optimal values. 